\title{
Evaluación de Propiedades Físicas y Mecánicas de Tableros OSB Utilizando Técnica de Ensayo No Destructiva
}

\author{
Elen Ap.Morales ${ }^{(1)}$, Francisco A. Rocco ${ }^{(2)}$, Maria F. Nascimento ${ }^{(2)}$ y Raquel Gonçalves $^{(3)}$ \\ (1) Universidad de São Paulo. Interunidades en Ciencias e Ingeniería de Materiales. Av. Trabalhador \\ Sãocarlense, 400, CEP: 13560590, Centro, São Carlos, SP-Brasil. (e-mail: emorales@sc.usp.br) \\ (2) Universidad de São Paulo. Escuela de Ingeniería de São Carlos, Departamento de Estructuras, \\ Laboratorio de Maderas y Estructuras de Maderas. Av. Trabalhador Sãocarlense, 400, \\ CEP: 13560590, Centro, São Carlos, SP-Brasil. (e-mails: frocco@sc.usp.br; fati@sc.usp.br) \\ (3) Universidad Estatal de Campinas. Fac. de Ingeniería Agrícola. Ciudad Universitaria Zeferino Vaz, \\ CP 6011, CEP: 13083875, Campinas, SP-Brasil. (e-mail: raquel@agr.unicamp.br)
}

\begin{abstract}
Resumen
Se ha investigado la eficiencia de la técnica de ultrasonido para determinar parámetros físicos y mecánicos de Tableros de Virutas Orientadas (OSB) de tipo comercial, fabricados en Brasil. Entre los productos derivados de la madera, el OSB es uno que ha sido crecientemente utilizado en subsistemas constructivos alrededor del mundo. Por otro lado, la aplicación de métodos no destructivos se ha tornado prometedora en materiales a base de madera. Para este trabajo se ensayaron tableros de uso estructural OSB/3, uno con $15 \mathrm{~mm}$ de espesor y el otro con $25 \mathrm{~mm}$. Los valores medios de velocidad obtenidos en tableros OSB en las direcciones longitudinal y transversal, así como la significativa correlación lineal entre algunas propiedades físico-mecánicas, valores de velocidades y de constantes dinámicas, sugieren la posibilidad de uso del método de ultrasonido en el control de calidad del OSB.
\end{abstract}

Palabras Claves: productos derivados de madera, métodos no destructivos, tablero de virutas orientadas, control de calidad, ultrasonido.

\section{Evaluating OSB Properties Using Nondestructive Technique}

\section{Abstract}

The efficiency of ultrasound techniques to estimate physical and mechanical parameters of commercial Oriented Strand Board (OSB) panels manufactured in Brazil, has been investigated. Among engineered wood products, the OSB is the one that has been increasingly used in building subsystems, all around the world. Also, nondestructive methods are frequently preferred to be applied to wood based materials. Structural OSB/3 panels were tested, being one $15 \mathrm{~mm}$ thick and the other $25 \mathrm{~mm}$ thick. The mean velocity values obtained in panels OSB in longitudinal and transversal directions, as well as the significant linear correlation between some physical and mechanical properties, the velocity and dynamic constants suggest the possibility of using ultrasound techniques in OSB quality control.

Keywords: engineered wood products, nondestructive methods, oriented strand board, quality control, ultra-sound 


\section{INTRODUCCIÓN}

Una de las tendencias más evidentes en la industria maderera es el crecimiento de la producción de los llamados tableros a base de madera. Entre esos productos, el que ha mostrado creciente aplicación mundialmente, en especial en la construcción civil, es el OSB (Tablero de Virutas Orientadas). Un cuidado especial que necesita urgentes inversiones de investigación, se refiere al desenvolvimiento de tecnología para permitir la evaluación más práctica de las propiedades mecánicas de los derivados de madera. Esta evaluación que también conduce al control de calidad de los tableros, normalmente se ha basado en procedimientos de muestras estadísticas, en las cuales partes de esos productos son testados destructivamente, en general horas o días después de su producción (Matos et al, 2000). Tal procedimiento demanda tiempo y material que no podrá ser utilizado después de testado. Además de esto, en caso que sean determinadas propiedades que no atiendan a los requisitos de calidad, no habrá como introducir modificaciones en la línea de producción para corregir el problema y reducir las pérdidas. El objetivo de este trabajo es investigar la eficiencia de técnicas no destructivas de ensayo (ultrasonidos) en la estimativa de parámetros físicos y mecánicos de tableros OSB comerciales de origen nacional.

Según la EN 300/2002 (Norma Portuguesa), el OSB (tablero de virutas largas y orientadas) es compuesto por varias camadas constituidas por virutas largas de madera de determinado formato y espesor, aglutinadas por una resina adhesiva. La Figura 1a muestra ejemplos de tableros OSB. Las virutas largas de madera de las camadas exteriores se encuentran alineadas y dispuestas paralelamente a lo largo o a lo ancho de la placa; y las de la o de las camadas interiores (centrales) pueden encontrarse orientadas aleatoria mente 0 alineadas, generalmente, en la dirección perpendicular a la de las virutas largas de madera de las camadas exteriores (Figura 1b). Estas virutas largas de madera, o "strands", son unidas unas a otras con resina a prueba de agua bajo calor y presión. (Youngquist, 1999)

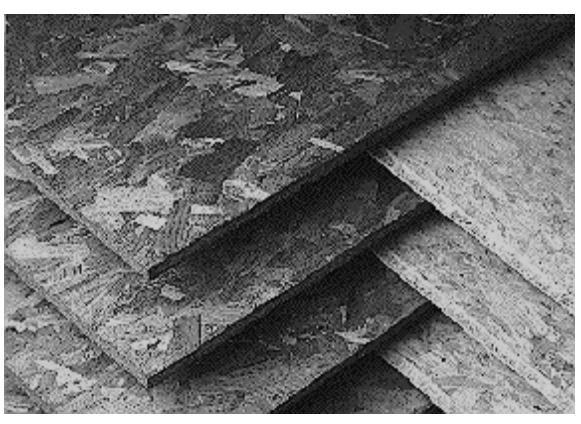

(a)

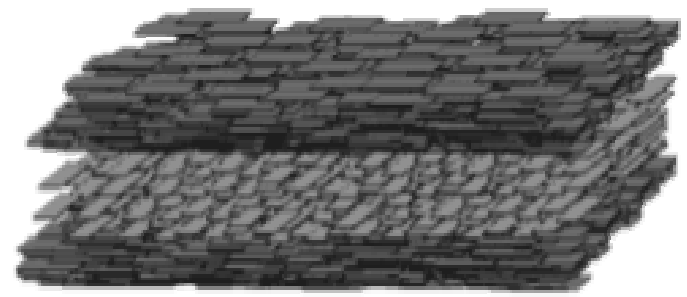

(b)

Fig. 1: Tableros OSB. (a) Ejemplos de tableros OSB y (b) Orientación de las camadas de los tableros OSB.

Entre los ensayos no destructivos pueden ser citados la clasificación visual (que es uno de los más ampliamente utilizados), testes químicos (para detectar defectos biológicos), "stress wave", ultrasonido, método de deflexión, teste de propiedades eléctricas, radiaciones gama, penetración de radiación y método de rayo $X$. De éstos los más utilizados para madera y sus derivados son: el "stress wave" y el ultrasonido (Bodig, 2001). La aplicación del método de ultrasonido consiste en el posicionamiento de dos transductores en el material a ser evaluado. La onda ultrasónica es inducida por uno de los transductores y captada por el otro, siendo la cuenta del tiempo, efectuada en microsegundos por el propio equipamiento de ultrasonido o por osciloscopio acoplado a éste. Los tiempos registrados son empleados en el cálculo de la velocidad de propagación de la onda (V) (Ecuación (1)).

$V=\frac{d}{t}$

Donde $\mathrm{V}=$ velocidad de propagación de la onda $(\mathrm{m} / \mathrm{s}), \mathrm{d}=$ distancia entre los transductores $(\mathrm{m})$ y $\mathrm{t}=$ tiempo de propagación de la onda (s) 
A partir del cálculo de $\mathrm{V}$ es posible calcular la constante dinámica (C) proveniente de la ecuación matemática basada en los mismos mecanismos que determinan las propiedades elásticas del material (Ecuación (2)):

$\mathrm{C}=\rho \cdot \mathrm{V}^{2} \cdot 10^{-6}$

Donde: $\mathrm{C}=$ constante elástica $(\mathrm{MPa}), \rho=$ densidad del material $\left(\mathrm{kg} / \mathrm{m}^{3}\right), \mathrm{V}=$ velocidad de propagación de la onda $(\mathrm{m} / \mathrm{s})$

Bekhta et al (2000) utilizaron la frecuencia de resonancia y la velocidad del sonido, dos métodos de caracterización no destructiva, con transductores de frecuencias 50, 100 y $200 \mathrm{kHz}$ en tableros de partículas, MDF y OSB, de producción industrial, de 16, 17 y 18mm de espesor, respectivamente, en las direcciones longitudinal y transversal a la dirección de la producción de lo tablero, así como en el espesor. Encontraron las siguientes fajas de velocidad en relación a la dirección de propagación de las ondas: 2271 a $3294 \mathrm{~m} / \mathrm{s}$ en la dirección longitudinal y 2118 a $2991 \mathrm{~m} / \mathrm{s}$ en la dirección transversal. Mendes et al (2002) realizaron estudios de evaluación no destructiva de propiedades mecánicas de tableros OSB producido con seis especies de Pinus spp y mezcla entre ellas (con y sin parafina), utilizando Stress Wave, buscando establecer correlación con las técnicas de evaluación convencionales. Encontraron bajos coeficientes de correlación entre los valores de MOE obtenidos en esos dos tipos de evaluación, pero alentaron para realizar más investigaciones en este tema con tableros OSB. Morales et al (2004) presentaron, de manera preliminar, el estudio de la eficiencia de la utilización del método no destructivo de ultrasonido para estimar propiedades mecánicas de tableros OSB. Fueron utilizados tableros de uso estructural OSB/3 de $15 \mathrm{~mm}$ de espesor y el equipamiento de ultrasonido de la marca Steinkamp, modelo BP7, con transductores de frecuencia igual a $45 \mathrm{kHz}$. Verificaron que en el espesor los tableros presentaron menor homogeneidad que al largo de las direcciones principales. Encontraron valores de velocidad en el espesor y en las direcciones longitudinal y transversal de dos tableros diferentes, $1116 \mathrm{~m} / \mathrm{s}, 3327 \mathrm{~m} / \mathrm{s}$ y $2674 \mathrm{~m} / \mathrm{s}$, con coeficientes de variación de $8 \%, 2 \%$ y $1 \%$, y bajos coeficientes de correlación entre los métodos de ensayo destructivo y de ultrasonido.

\section{MATERIALES Y MÉTODOS}

Fueron ensayados tableros de uso estructural OSB/3 de origen nacional, uno con 15mm de espesor y lo otro con $25 \mathrm{~mm}$. Los mismos son constituidos por tres camadas, siendo las externas con virutas orientadas paralelas entre sí, en la dirección del largo de lo tablero, y la camada interior con las virutas dispuestas en la dirección perpendicular a los de las camadas externas. Inicialmente los tableros fueron demarcados en sus ancho y largo, de 50 en $50 \mathrm{~mm}$ y fueron diseñados provetes para los ensayos físicos y mecánicos del humedad, densidad, hinchamiento en espesor, absorción del agua, módulos de elasticidad en las direcciones longitudinal y transversal, módulo de resistencia en las direcciones longitudinal y transversal a flexión y resistencia a la tracción perpendicular a las caras de los tableros (representados respectivamente por $H, \rho, G_{t}, A_{m}, E_{m / /}, E_{m \perp}, f_{m / /}, f_{m \perp}$ y $A l$ ), siguiendo las prescripciones de la EN 326-1/1994. Se tomó el criterio de tener como mínimo 30 provetes para cada tipo de ensayo, para obtener grandes muestras (Figuras 2a e b).

Después fueron realizados los ensayos no destructivos con el equipamiento de ultrasonido en los tableros, en el Laboratorio de Acustoelástica, de la Facultad de ingeniería Agrícola (FEAGRI) de la Universidad Estatal de Campinas (UNICAMP). El aparato utilizado fue el STEINKAMP, modelo BP7 con transductores planos, de $45 \mathrm{kHz}$ de frecuencia, con gel medicinal como acoplante. Se procedió a la medición del tiempo y propagación de la onda a lo largo de las direcciones longitudinal y transversal de los tableros, así como en el espesor de los mismos, en los lugares que se refieren a los provetes diseñados (Figuras $3 a$, b y c), para la determinación de los valores de las velocidades y subsecuentes correlaciones con los valores obtenidos en los ensayos físico y mecánicos realizados posteriormente. Esas mismas correlaciones fueron realizadas con los valores de C. 


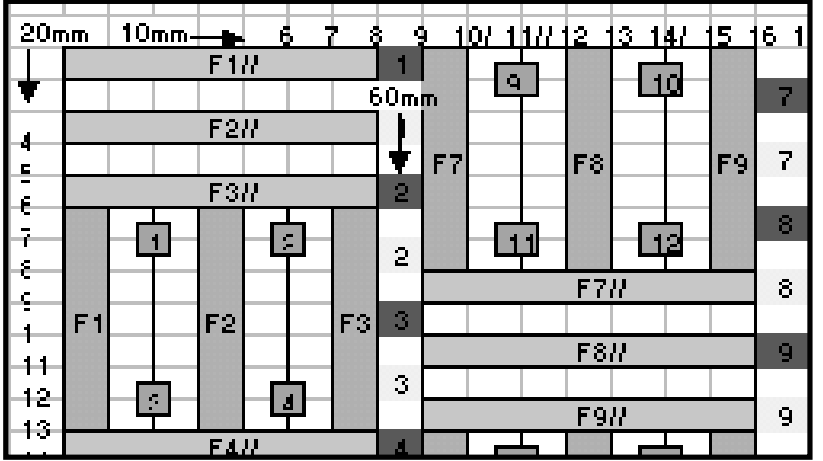

(a)

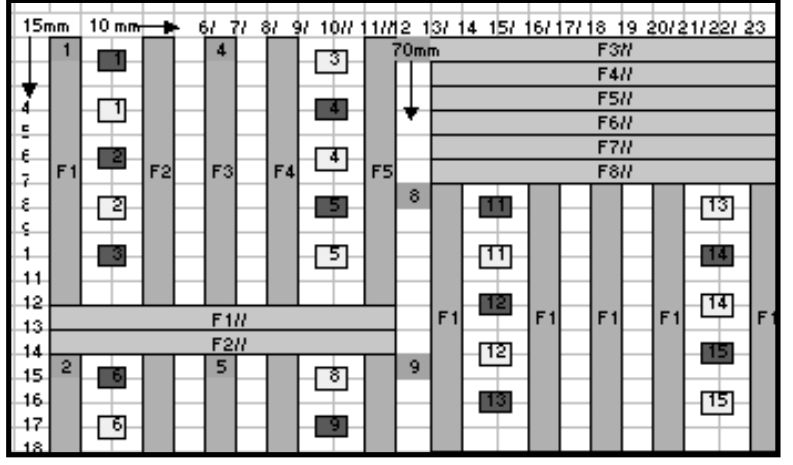

(b)

Fig.2: Demarcación de los tableros: (a) Espesor 15mm y (b) Espesor 25mm.

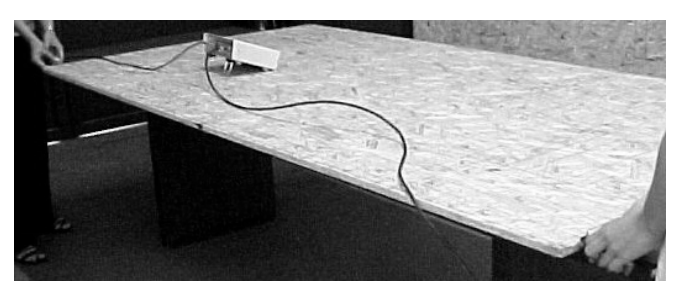

(a)

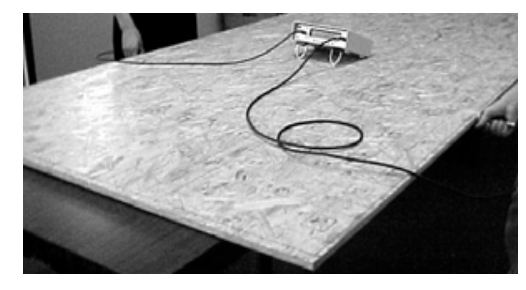

(b)

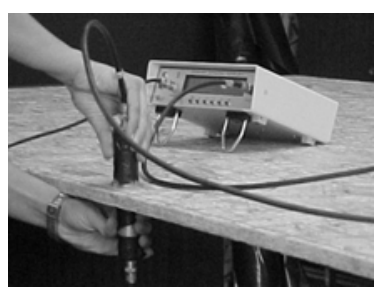

(c)

Fig. 3: Mediciones de tiempo de propagación con el aparato de ultrasonido: (a) Dirección longitudinal, (b) Dirección transversal y (c) Espesor de lo tablero.

Tabla 1: Propiedades físicas y mecánicas de los tableros OSB. $(\mathrm{t}=$ espesor $)$

\begin{tabular}{ccccccccccc} 
& $\begin{array}{c}\mathrm{t} \\
(\mathrm{mm})\end{array}$ & $\begin{array}{c}\mathrm{H} \\
(\%)\end{array}$ & $\begin{array}{c}\rho \\
\left(\mathrm{kg} / \mathrm{m}^{3}\right)\end{array}$ & $\begin{array}{c}\mathrm{G}_{\mathrm{t}} \\
(\%)\end{array}$ & $\begin{array}{c}\mathrm{A}_{\mathrm{m}} \\
(\%)\end{array}$ & $\begin{array}{c}\mathrm{E}_{\mathrm{m} / /} \\
(\mathrm{MPa})\end{array}$ & $\begin{array}{c}\mathrm{E}_{\mathrm{m} \perp} \\
(\mathrm{MPa})\end{array}$ & $\begin{array}{c}\mathrm{f}_{\mathrm{m} / /} \\
(\mathrm{MPa})\end{array}$ & $\begin{array}{c}\mathrm{f}_{\mathrm{m} \perp} \\
(\mathrm{MPa})\end{array}$ & $\begin{array}{c}\mathrm{Al} \\
(\mathrm{MPa})\end{array}$ \\
\hline $\bar{x}$ & & 9 & 595 & 15 & 71 & 4142 & 1652 & 25 & 14 & 0,44 \\
$\mathrm{~s}$ & 15 & 0,12 & 44 & 3 & 9 & 590 & 231 & 6 & 2 & 0,13 \\
$\mathrm{CV}(\%)$ & & 1 & 7 & 21 & 13 & 14 & 14 & 25 & 18 & 30 \\
\hline $\bar{x}$ & & 9 & 595 & 19 & 87 & 4553 & 1817 & 26 & 13 & 0,18 \\
$\mathrm{~s}$ & 25 & 0,17 & 21 & 1 & 6 & 680 & 132 & 4 & 2 & 0,08 \\
$\mathrm{CV}(\%)$ & & 2 & 4 & 7 & 7 & 15 & 7 & 15 & 13 & 46 \\
\hline
\end{tabular}

\section{RESULTADOS}

Los resultados obtenidos en los ensayos de propiedades físicas y mecánicas realizados son presentados en la Tabla 1.

Los valores referidos a las velocidades y constantes dinámicas obtenidas por mediciones del tiempo de propagación, por el aparato de ultrasonido, en el espesor de las placas, en los locales correspondientes a los provetes de tracción perpendicular a las caras $\left(\mathrm{V}_{\mathrm{Al}}\right.$ y $\left.\mathrm{C}_{\mathrm{Al}}\right)$, densidad $\left(\mathrm{V}_{\mathrm{d}}\right.$ y $\left.\mathrm{C}_{\mathrm{d}}\right)$, hinchamiento en espesor $\left(V_{1}\right.$ y $\left.C_{1}\right)$ y absorción de agua $\left(V_{A I}\right.$ y $\left.C_{A I}\right)$ están presentados en la Tabla 2. Los valores referentes a las velocidades y constantes dinámicas obtenidas por mediciones de tiempo en las direcciones longitudinal $(/ /)$ y transversal $(\perp)$ de las placas están presentados en el Tabla 3 . Las ecuaciones de regresión entre las propiedades estáticas y dinámicas (espesores $15 \mathrm{~mm} x$ $25 \mathrm{~mm})$, y sus referentes valores de coeficientes de determinación $\left(R^{2}\right)$ y de correlación $(R)$, están presentados en el Tabla 4, donde $F_{\max }$ representa la fuerza de ruptura de los provetes en el ensayo de flexión. 
Tabla 2: Velocidades y constantes dinámicas calculadas a partir de los tiempos medidos en el espesor de los tableros

\begin{tabular}{cccccccccc}
\cline { 2 - 9 } & $\begin{array}{c}\mathrm{t} \\
(\mathrm{mm})\end{array}$ & $\begin{array}{c}\mathrm{V}_{\mathrm{Al}} \\
(\mathrm{m} / \mathrm{s})\end{array}$ & $\begin{array}{c}\mathrm{V}_{\mathrm{d}} \\
(\mathrm{m} / \mathrm{s})\end{array}$ & $\begin{array}{c}\mathrm{V}_{\mathrm{l}} \\
(\mathrm{m} / \mathrm{s})\end{array}$ & $\begin{array}{c}\mathrm{V}_{\mathrm{A}} \\
(\mathrm{m} / \mathrm{s})\end{array}$ & $\begin{array}{c}\mathrm{C}_{\mathrm{Al}} \\
(\mathrm{MPa})\end{array}$ & $\begin{array}{c}\mathrm{C}_{\mathrm{d}} \\
(\mathrm{MPa})\end{array}$ & $\begin{array}{c}\mathrm{C}_{\mathrm{I}} \\
(\mathrm{MPa})\end{array}$ & $\begin{array}{c}\mathrm{C}_{\mathrm{A}} \\
(\mathrm{MPa})\end{array}$ \\
\hline $\bar{x}$ & & 1086 & 1091 & 1102 & 1104 & 706 & 722 & 726 & 728 \\
$\mathrm{~s}$ & 15 & 81 & 108 & 76 & 76 & 104 & 175 & 99 & 100 \\
$\mathrm{CV}(\%)$ & & 7 & 10 & 7 & 7 & 15 & 24 & 14 & 14 \\
\hline $\bar{x}$ & & 963 & 965 & 966 & 966 & 554 & 555 & 557 & 556 \\
$\mathrm{~s}$ & 25 & 71 & 50 & 55 & 55 & 80 & 66 & 62 & 62 \\
$\mathrm{CV}(\%)$ & & 7 & 5 & 6 & 6 & 15 & 12 & 11 & 11 \\
\hline
\end{tabular}

Tabla 3: Velocidades y constantes dinámicas calculadas a partir de los tiempos medidos en las direcciones longitudinal y transversal de los tableros

\begin{tabular}{cccccc} 
& $\mathrm{T}(\mathrm{mm})$ & $\mathrm{V}_{/ /}(\mathrm{Mpa})$ & $\mathrm{V}_{\perp}(\mathrm{MPa})$ & $\mathrm{C}_{/ /}(\mathrm{MPa})$ & $\mathrm{C}_{\perp}(\mathrm{MPa})$ \\
\hline $\bar{x}$ & & 3039 & 2575 & 5498 & 3946 \\
$\mathrm{~s}$ & 15 & 21 & 28 & 76 & 86 \\
$\mathrm{CV}(\%)$ & & 1 & 1 & 1 & 2 \\
\hline $\bar{x}$ & & 3216 & 2658 & 6150 & 4201 \\
$\mathrm{~s}$ & 25 & 44 & 24 & 166 & 76 \\
$\mathrm{CV}(\%)$ & & 1 & 1 & 3 & 2 \\
\hline
\end{tabular}

Tabla 4: Ecuaciones de regresión entre las propiedades estáticas y dinámicas y sus referentes valores del $R^{2}$ y $R$

\begin{tabular}{ccc|ccc}
\hline $\mathrm{R}^{2}$ & $\mathrm{R}$ & Ecuaciones & $\mathrm{R}^{2}$ & $\mathrm{R}$ & Ecuaciones \\
\hline 0,09 & 0,30 & $\mathrm{f}_{\mathrm{m} / /}=0,0169 \mathrm{~V}_{/ /}-27,095$ & 0,15 & 0,39 & $\mathrm{~d}=0,1286 \mathrm{~V}_{\mathrm{d}}+460,93$ \\
0,00 & 0,00 & $\mathrm{f}_{\mathrm{m} \perp}=-0,0011 \mathrm{~V}_{\perp}+16,297$ & 0,38 & 0,62 & $\mathrm{Al}=0,0011 \mathrm{~V}_{\mathrm{Al}}-0,7668$ \\
0,74 & 0,86 & $\mathrm{~F}_{\max / /}=2,693 \mathrm{~V}_{/ /}-7523,4$ & 0,38 & 0,62 & $\mathrm{Al}=0,0009 \mathrm{C}_{\mathrm{Al}}-0,234$ \\
0,61 & 0,78 & $\mathrm{~F}_{\max \perp}=1,9916 \mathrm{~V}_{\perp}-4753,7$ & 0,23 & 0,48 & $\mathrm{G}_{\mathrm{t}}=-0,0168 \mathrm{~V}_{\mathrm{Gt}}+34,475$ \\
0,24 & 0,49 & $\mathrm{E}_{\mathrm{m} / /}=0,9531 \mathrm{C}_{/ /}-1189,8$ & 0,24 & 0,49 & $\mathrm{G}_{\mathrm{t}}=-0,0137 \mathrm{C}_{\mathrm{Gt}}+25,887$ \\
0,23 & 0,48 & $\mathrm{E}_{\mathrm{m} \perp}=0,6698 \mathrm{C}_{\perp}-992,74$ & 0,55 & 0,74 & $\mathrm{~A}_{\mathrm{m}}=-0,0856 \mathrm{~V}_{\mathrm{Am}}+168,01$ \\
& & & 0,55 & 0,74 & $\mathrm{~A}_{\mathrm{m}}=-0,0686 \mathrm{C}_{\mathrm{Am}}+123,53$ \\
\hline
\end{tabular}

\section{DISCUSIÓN}

Los valores obtenidos en los ensayos de caracterización física y mecánica de los tableros, alcanzaron, y hasta superaron los valores estipulados por el documento normativo EN 300/2002, para tableros OSB/3, con excepción de los valores de: hinchamiento en espesor (24h) y tracción perpendicular a las caras de lo tablero de $25 \mathrm{~mm}$ de espesor. Los mayores coeficientes de variación fueron obtenidos entre los valores de resistencia de la tracción perpendicular a las caras. Los valores de las velocidades en el espesor y en las direcciones longitudinal y transversal de dos tableros se mostraron diferentes, como los obtenidos por Morales (2004), con coeficientes de variación entre 1\% y $10 \%$, valores considerados muy bajos. Esta diferencia también sucedió para los valores de las constantes dinámicas en el espesor y en las direcciones longitudinal y transversal de los tableros.

Los valores de velocidades en las direcciones longitudinal y transversal están de acuerdo con los encontrados por Bekhta et al (2002). Los valores de las velocidades medidas en el espesor fueron mayores para lo tablero de espesor de $15 \mathrm{~mm}$ que para lo de $25 \mathrm{~mm}$, posiblemente porque, siendo ese último de mayor espesor, presenta mayor atenuación. 
Nuevamente, como obtenido por Morales (2004), los bajos coeficientes de correlación determinados en ambos métodos, destructivo convencional y de ultrasonido, muestran la homogeneidad de los tableros OSB. Debido a esa homogeneidad los intervalos de variación de esas propiedades solamente permitieron la obtención de modelos de correlaciones lineares significativos entre los valores de $F_{\max / /}, F_{\max \perp}$ y $A_{m}$ y los valores de $V_{/ /}, V_{\perp}, V_{A m}$ y $C_{A m}$, que presentarán valores de $R^{2}$ mayores que 0,50 .

\section{CONCLUSIONES}

a) Los bajos coeficientes de determinación obtenidos por los dos métodos de ensayo muestran la homogeneidad de los tableros OSB. Por medio del ensayo utilizando ultrasonido fue posible verificar que, en el espesor, los tableros presentaron mayor heterogeneidad que a lo largo de las direcciones principales.

b) Los valores de velocidades y constantes elásticas diferenciados en las direcciones longitudinal y transversal muestran que es posible detectar la variación de características mecánicas de placas OSB utilizando el ultrasonido.

c) Los valores de velocidades encontrados en las direcciones longitudinal y transversal de los tableros están de acuerdo con los ya encontrados por otros autores.

d) Los resultados obtenidos, bien como los modelos de correlaciones lineares significativos entre los valores de $F_{\max / /,} F_{\max \perp}$ y $A_{m}$ y los valores de $V_{/ /}, V_{\perp}, V_{A m}$ y $C_{A m}$, sugieren la posibilidad de utilización del método de ultrasonido en el control de calidad de parámetros mecánicos de tableros OSB comerciales y, por lo tanto, la continuidad del estudio.

\section{AGRADECIMIENTOS}

A la CAPES por el apoyo financiero a esta pesquisa.

\section{REFERENCIAS}

Bekhta, P. A., Niemz, P. y Kucera, L., "The Study of Sound Propagation in the Wood-Based Composite Materials", $12^{\text {th }}$ Symposium on Nondestructive Testing of Wood, Western Hungary Sopron (2000).

Bodig, J. "The process of NDE research for wood and wood composites", The e-Journal of Nondestructive testing, ISSN: 1435-4934, 6 (3), 2001 (fecha de consulta Septiembre 22, 2002), acesso libre: http://www.ndt.net/index.html.

EN 300, European Standard, Aglomerado de Partículas de Madeira Longas e Orientadas (OSB), 627, Portugal (2002).

Youngquist, J. A. "Wood-based Composites and Panel Products", In Wood handbook - Wood as an engineering material, pp (10)1-(10)30. Gen. Tech. Rep. FPL - GTR - 113, Madison (1999).

Matos, J. L. M., Keinert Jr., S. y Rosa, G. M., "Métodos de Emissão Acústica para Determinação Não Destrutiva de Propriedades de Painéis Compensados de Madeira.", $5^{\text {th }}$ Encontro Brasileiro de Madeiras e em Estruturas de Madeira, São Carlos, SP - Brasil, Julio 12-14 (2000).

Mendes, L. M., Matos, J. L. M., Iwakiri, S., Mori, F. A. y Truguilho, P. F., "Uso de Métodos de Emissão Acústica para Determinação Não-Destrutiva de Propriedades de Painéis OSB (Orientd Strand Board)", $2^{\text {th }}$ Congresso Ibero-Americano de Pesquisa e Desenvolvimento de Produtos Florestais, Curitiba Brasil, Septiembre 9-13 (2002).

Morales, E. A. M., Rocco Lahr, F. A., Nascimento, M. F. y Gonçalves, R., "Estimativa de Propriedades Mecânicas de Painéis OSB (Oriented Strand Board) Através de Uso de Ultra-Som", $10^{\text {th }}$ Encontro Brasileiro em Madeiras e Estruturas de Madeiras, Cuiabá - Brasil, Julio 27-29 (2004). 